The effect of behavi or al preferences on ski II acqui si ti on i n determing unspecified, sui tabl e acti on patterns to control humanoi d robots

\begin{tabular}{|l|l|}
\hline 著者 & Takayana Tai ki , Wat anabe Tet suy ou \\
\hline $\begin{array}{l}\text { j our nal or } \\
\text { publ i cat i on ti t l e }\end{array}$ & $\begin{array}{l}\text { Proceedi ngs of I nt er nat i onal Conf er ence of the } \\
\text { I EEE Engi neer i ng i n Nedi ci ne and Bi ol ogy } \\
\text { Soci et y ( EMBC) }\end{array}$ \\
\hline vol une & 2015- Novenber \\
\hline page range & $7586-7589$ \\
\hline year & 2015- 11- 04 \\
\hline URL & ht t p: //hdl . handl e. net /2297/46075 \\
\hline
\end{tabular}




\title{
The effect of behavioral preferences on skill acquisition in determining unspecified, suitable action patterns to control humanoid robots
}

\author{
Taiki Takayama and Tetsuyou Watanabe, Member, IEEE
}

\begin{abstract}
This research investigated the effect of behavioral preferences on learning efficiency when attempting to determine unspecified, but suitable action sequences for unfamiliar tasks. The goal of this research was to develop a skill acquisition support system for the elderly to aid them in using unfamiliar IT products, particularly those of welfare systems. Here, behavioral preference is defined as the type of action sequences that people would prefer to adopt for completing unfamiliar tasks. To achieve this goal, this research investigated the action sequences of participants when they attempt to control the posture of an unfamiliar humanoid robot with an unfamiliar controller. The participants were assigned the task of making the humanoid stand on one foot. Machine-learning-based methods were presented for analyzing the behavioral preferences. The analysis results indicate that participants having behavioral preferences of adopting random action sequences can complete the task in a much shorter time, compared to participants having a behavioral preference of adopting action sequences similar to those of previous actions.
\end{abstract}

\section{INTRODUCTION}

This research investigated the behavioral preferences in the learning process of participants when they attempt to determine unspecified, but suitable action sequences for completing unfamiliar tasks: controlling the posture of humanoid robots that are expected to help the elderly in the future. In this study, "unspecified" indicates that the number of suitable sequences is infinite. For example, when operating humanoid robots, different command sequences are available for achieving the same final posture. It is necessary to determine the types of action sequences that people prefer to adopt during the learning process. Some people tend to repeat similar action sequences while other people tend to adopt different action sequences. Behavioral preference is defined as the pattern of a selected action sequence. This research investigated the effect of behavioral preferences on learning time and efficiency.

This research was motivated by the need for elderly people to acquire skills that will help them use IT products. There are numerous welfare and healthcare systems that require the use of smart phones and tablet PCs. They are useful and have high potential in supporting societies with aging populations. The elderly, as well as middle-aged people who support the elderly, therefore need to learn to use IT products such as smartphones and tablet PCs. Current IT products come with little or no instructions for use, and therefore, the user has to determine an unspecified, but suitable action sequence for completing a

*Research partly supported by JSPS Kakenhi 26540143.

T. Takayama is with the Graduated school of Natural science and Technology, Kanazawa University, Kakuma-machi, Kanazawa, 9201192, Japan (e-mail: t.taiki@stu.kanazawa-u.ac.jp). desired task on his/her own. However, it is difficult for the elderly and middle-aged people to learn these required IT skills, as reported in [1], resulting in the so-called digital divide. In order to make the best use of IT products, including those of welfare systems, systems that facilitate skill acquisition may be required. However, it is difficult to develop such a support system because a suitable sequence, which the user has to determine, is unspecified. First, this research investigated the processes involved in learning or determining a suitable sequence in the operation of IT products. Controlling the posture of a humanoid robot was set as the target operation, as this task would be unfamiliar to all participants, and they would need to acquire this skill of controlling the posture. A significant outcome of this study is that a humanoid robot could one day help or support the elderly to carry out everyday tasks. Machine-learning based methods were also presented in order to extract the behavioral preferences obtained from experimental results; these preferences were then analyzed.

Plenty of research has been conducted on skill acquisition. One typical example of skill acquisition for determining unspecified sequences for task completion is found in the production of traditional handicrafts. To facilitate the transfer of skills from an expert to a beginner (next generation), several supporting systems were developed $[2,3]$. If a person learning Japanese calligraphy takes a bamboo brush attached to a robot, the robot moves along a trajectory (sequence) recorded with an expert; the person learning can then follow the expert's trajectory. In those studies [2, 3], the key to dealing with unspecified sequences was to specify that the suitable trajectory was that of the expert's, with the assumption that the expert's trajectory is perfect. However, there is no guarantee that following the expert's trajectory/sequence would be effective for beginners to acquiring that certain skill. There is a support system that displays the expert's eye movements [4]. The eye movement of the expert could provide hints for task completion. It would be useful if the sequence for the task were specified. Another example of skill acquisition for determining unspecified sequences for tasks is found in sports. In the field of sports engineering, effective methods for athletes or experts to polish their skills have been studied $[5,6]$. However, the target for acquisition is different, and hence, the methods employed in that field cannot be used in this study. In the fields of neuroscience and psychology, a large amount of research on skill acquisition has been conducted. Random patterns can provide better learning and retention [7]. It is associated with the concept of the contextual interference effect in psychology

T. Watanabe is with the Institute of Science and Engineering, Kanazawa University, Kakuma-machi, Kanazawa, 9201192 Japan (e-mail: tewatanabe@ieee.org) 
[8]. Some learning mechanisms [9] such as motor chunking $[10,11]$ and associative learning $[12,13]$ have been presented. However, the sequences to be learned were specified. Therefore, another type of study is required to understand the learning process when acquiring skills associated with unspecified sequences.

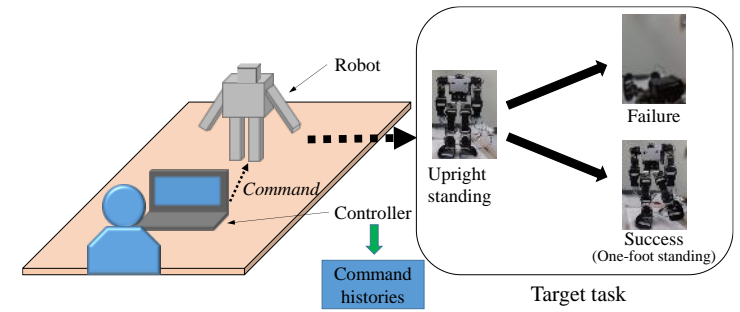

Figure 1. Schematic view of experimental setup with target task: controlling a humanoid to shift from an upright standing posture (standing with both feet on the ground) to a one-foot standing posture

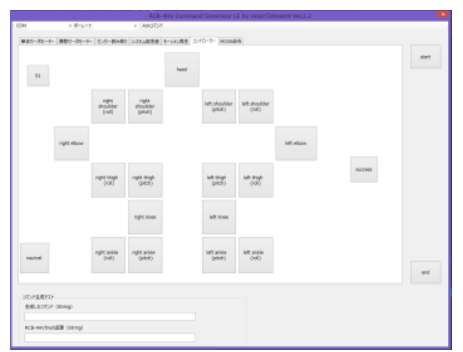

Figure 2. Controller display on the tablet PC

TABLE I. ASSOCIATION BETWEEN BUTTON NUMBERS AND JOINTS WITH THEIR ROTATIONAL DIRECTION (THE INDIVIDUAL JOINTS FOR UPPER BODY ARE NOT SHOWN BECAUSE THEY WERE NOT USED)

\begin{tabular}{|l|c|c|}
\hline \multirow{2}{*}{ Joint } & \multicolumn{2}{|c|}{ Button number } \\
\cline { 2 - 3 } & Positive rotation & $\begin{array}{c}\text { Negative/inverse } \\
\text { rotation }\end{array}$ \\
\hline Joints for upper body & \multicolumn{2}{|c|}{$1-14$} \\
\hline Right thigh (roll) & 15 & 16 \\
\hline Right thigh (pitch) & 17 & 18 \\
\hline Left thigh (pitch) & 19 & 20 \\
\hline Left thigh (roll) & 21 & 22 \\
\hline Right knee & 23 & 24 \\
\hline Left knee & 25 & 26 \\
\hline Right ankle (roll) & 27 & 28 \\
\hline Right ankle (pitch) & 29 & 30 \\
\hline Left ankle (pitch) & 31 & 32 \\
\hline Left ankle (roll) & 33 & 34 \\
\hline
\end{tabular}

\section{Methodology}

\section{A. Participants}

The participants who took part in the experiment were eleven healthy students consisting of four males and seven females (aged between 18 and 22 years, with an average age of 20.5 years). The experiment was approved by the Medical Ethics Committee of Kanazawa University.

\section{B. Task}

The task was to control a humanoid robot, to make it shift from its initial upright standing posture to a single-foot standing posture, as shown in Fig. 1. A single-foot standing posture is defined as being able to stand with either foot off the ground, without falling under a steady-state condition. If a participant succeeded in achieving a single-foot standing posture three times, the experiment was stopped. The experiment was also stopped if one hour had elapsed without completion of the task.

The operation of a humanoid robot was used as the target task in this study; this operation is unfamiliar to the general public. Therefore, the participants of this study would not know how to perform the operation in advance, and they would attempt to determine unspecified and suitable action sequences for completing this unfamiliar task. In addition, the controller for the robot, which was a self-made application written for tablet PCs, was also unfamiliar to the participants.

\section{Apparatus}

Fig. 1 shows the schematic view of the experimental setup. The humanoid robot was a Kondo Kagaku KHR-3HV, Ver. 2. The tablet PC was a Sony VAIO Duo 11. The participants controlled the humanoid robot through a controller displayed on the tablet PC. The controller was created in Microsoft Visual Studio 2012 based on the Rcb4 library presented by Kondo Kagaku. The program for the controller was written in the Visual C\# language.

The controller is shown in Fig. 2. If a participant touches or pushes the button displayed on the touch screen, the corresponding motor rotates at a constant angle of 72/23 degrees. The name of the associated joint is displayed on the button. The button numbers were used to simplify the recording and analysis. Table 1 lists the association between the button numbers and joints with their rotational direction. If the button is touched or pushed, the associated number as well as the time it was pushed is recorded in the command history.

\section{Procedure}

The task (mentioned above) and the operation of the controller were explained to the participants. The association between the buttons and the joints with rotational direction was explained to the participants by illustrating how the robot moved in response to the commands provided. In a preliminary experiment, it took a very long time if a participant attempted to control all joints (at least $1 \mathrm{~h}$ was required even when experts tried). The participants were then asked to control only the joints of the lower body.

The participants carried out the robot operation. Every trial started from an initial state where the robot had an upright standing posture. The participant then tried to achieve the required single-foot standing posture. If the robot fell down or the participants determined that the robot could not attain the one-foot standing posture (for example, in cases where the robot had postures indicating that it was about to fall), the trial was stopped and designated a failure. After every trial, we asked the participants to push button 35 if the trial was a failure and push button 36 if the trial was a success. The experiments were completed or stopped when the participant succeeded with the trials three times or when one hour had elapsed.

\section{RESULTS}

\section{A. Total operation time}

Fig. 3 shows the total operation time for each participant, which is the total time required for successful completion of the trial three times. In the figure, "Par." refers to participant. Note that the total operation time for Par. 6 and Par. 8 was one 
hour. In the case of Par. 6, none of the trials were successful, and in the case of Par. 8, only one trial was successful in the stipulated 1-hour limit.

\section{B. Command history}

Fig. 4 shows part of the command history for Par. 2 as an example. The horizontal axis shows the number of commands that the participant executed, and the vertical axis shows the button number associated with a joint with rotational direction. Note that button 35 indicates failure and button 36 indicates success. Using these commands, the operation can be divided into each trial, as seen in Fig. 4.

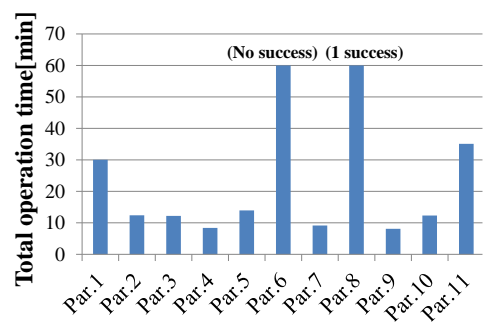

Figure 3. Total operation time for each participant: Par. stands for participant.
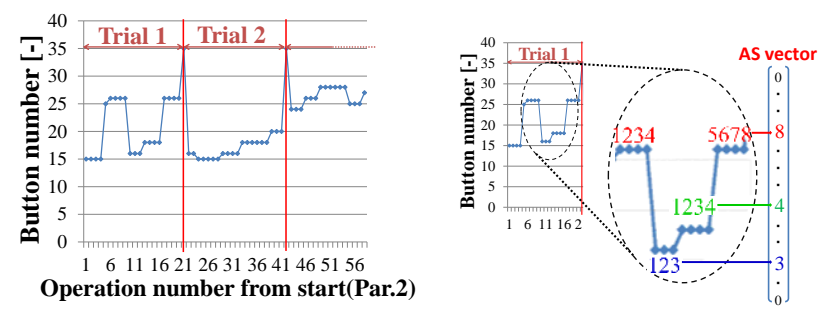

Figure 4. Command history for Par.2 Figure 5. Example representation of trial data as a AS vector

\section{MACHINE LEARNING BASED BEHAVIOR PREFERENCE ANALYSIS}

The presence of many similar action sequences indicates that the participant preferred those certain action sequences. Therefore, in order to observe the behavioral preference at each trial, we studied the similarities among action sequences. Here, machine learning [14]-based methods for analyzing the similarities (behavioral preferences) are presented.

\section{A. Similarity among action sequences}

Each trial was represented by the vector

$$
\boldsymbol{x}=\left[x_{1}, x_{2}, \cdots x_{34}\right]^{\mathrm{T}} \in R^{34}
$$

where $x_{k}$ represents the number of times button $k$ was pushed during the trial. We call the vector $\boldsymbol{x}$ an AS (action sequence) vector, as shown in Fig. 5. The final posture of the robot did not depend on the order a button was pushed, but rather on the number of times a button was pushed. For example, the following two sequences resulted in a similar rotation angle for the knee joint: (1) 4 times pushing button for knee joint, pushing button for other joints, 4 times pushing button for knee joint; and (2) 8 times pushing button for knee joint. Therefore, the command order information was ignored for this analysis. In order to better understand how the participants control the joints, the numbers associated with the positive and negative/inverse rotations for the same joint were separately treated. For example, if the participant pushed the button numbers associated with both positive and negative/inverse rotations, it indicates that the participant tried to control the joint precisely. On the other hand, if the participant pushed the button number associated with either one rotation, it indicates rough or brave control.

By utilizing the AS vector $\boldsymbol{x}$, dissimilarities between action sequences were analyzed. Let $N$ be the number of trials for a certain participant. Let $\boldsymbol{x}_{\mathrm{i}}$ be $\boldsymbol{x}$ for the ith trial. The dissimilarity between $\boldsymbol{x}_{\mathrm{i}}$ and $\boldsymbol{x}_{\mathrm{j}}$ can thus be defined by

$$
d_{i j}=\left|x_{\mathrm{i}}-\boldsymbol{x}_{\mathrm{j}}\right|(i \neq j)(i, j=1, \cdots N)
$$

$d_{i j}$ was calculated for all pairs.

$\mathrm{k}$-means clustering $(\mathrm{k}=2)$ was performed by setting the mean and median values for $d_{i j}$ as feature values. Note that median value was considered for taking distribution form into account. Fig. 6 shows clustered groups: Groups 1 and 2. Group 1 has relatively small median and mean values of dissimilarity, which indicates that the participants belonging to Group 1 performed or repeated similar action sequences to determine a suitable sequence for completing the given task. On the other hand, the participants belonging to Group 2 performed relatively randomized action sequences. These results are due to behavioral preferences.

Fig. 7 shows the mean total operation time with SD. for each group. It is observed that the participants belonging to Group 2 completed the sequence in a short time, whereas the participants belonging to Group 1 required a long time. However, there was not a statistically significant difference between the groups when applying Welch's t-test. In order to determine the reason for this, similarities to suitable sequences for completion are focused on and investigated in the next subsection.
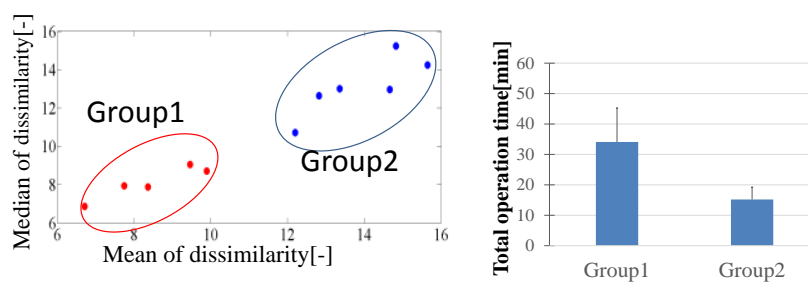

Figure 6. k-means clustering $(\mathrm{k}=2)$ result for dissimilarity between each trial

Figure 7. Mean total operation time for clustered groups with SD.

\section{B. Similarity between suitable sequence for completion and} trial sequences for participants in Group 1

The similarity between suitable sequences for completion and the trial sequences for the participants in Group 1 was investigated. The number of suitable sequences is more than one. Therefore, the set of suitable sequences should be compared with action sequences of the trials. The method based on nearest neighborhood algorithm [14] was then used for the analysis. Because the suitable sequence is obtained at the final posture, the final posture vector $\boldsymbol{x}_{f}$ was used instead of the AS vector $\boldsymbol{x}$ where

$$
\boldsymbol{x}_{f}=\left[x_{f 1}, x_{f 2}, \cdots x_{\mathrm{f} 17}\right]^{\mathrm{T}} \in R^{17}
$$




$$
x_{f i}=x_{2 i-1}-x_{2 i}
$$

Let $S_{\mathrm{s}}$ be the set of final postures and their laterally inverted postures for the successful trials for all participants. Because the robot is bilaterally symmetric, postures laterally inverted to those under stable conditions are also considered stable. Let $S_{\mathrm{t}_{i}}$ be the set of $\boldsymbol{x}_{f}$ vectors corresponding to all AS vectors for the $i$ th participant. The dissimilarity between $\boldsymbol{x}_{\mathrm{f}_{\mathrm{ij}}} \in S_{\mathrm{t}_{i}}$ (where $\boldsymbol{x}_{\mathrm{f}_{\mathrm{ij}}}$ corresponds to the $j$ th AS vector for the $i$ th participant) and the set of stable final postures $S_{\mathrm{s}}$ was then calculated as follows:

$$
\rho_{i j}=\min _{\boldsymbol{x}_{\mathrm{f}_{\mathrm{k}}} \in S_{\mathrm{s}}}\left|\boldsymbol{x}_{\mathrm{f}_{\mathrm{ij}}}-\boldsymbol{x}_{\mathrm{f}_{\mathrm{k}}}\right|
$$

where $\boldsymbol{x}_{\mathrm{f}_{\mathrm{k}}} \in S_{\mathrm{s}}$ is the $k$ th $\boldsymbol{x}_{f}$ in $S_{\mathrm{s}}$. The k-means clustering $(\mathrm{K}=2)$ was conducted by setting the following mean and median maximum values of $\rho_{i j}$ for every participant as feature values:

$$
\rho_{\text {mean }_{i}}=\operatorname{mean}_{\mathrm{j}} \rho_{i j}, \quad \rho_{\max _{i}}=\max _{\mathrm{j}} \rho_{i j}
$$

Here we saw how far from stable one-foot standing postures participant tried to control the robot to, from the viewpoint of "maximally" and "on average". Fig. 8 shows the clustering result, where Group 1A and Group 1B are the two clustered groups. There are two participants in Group 1. The participants in Group 1A repeated similar action sequences that were quite different from suitable sequences, whereas the participants in Group 1B repeated similar action sequences that were similar to suitable sequences. Fig. 9 shows the mean total operation time with SD. for Group 1A, Group 1B, and Group 2. The task completion times for Group 1A were long, whereas those for Group 1B times were short, shorter than Group 2 even. Statistically significant differences were observed between Group 1A and Group 2 and between Group $1 \mathrm{~A}$ and Group 1B, when applying Tukey's test. The reason for the non-significant differences between Group 1 and Group 2 is considered to be due to the large difference between Group $1 \mathrm{~A}$ and Group $1 \mathrm{~B}$.

\section{DISCUSSION}

This research investigated the effect of behavioral preferences on learning time and efficiency, with the aim of developing systems to facilitate skill acquisition for the elderly to aid them in using unfamiliar IT products, particularly those of welfare care systems. To achieve this goal, the learning process when participants tried to control the posture of an unfamiliar humanoid robot was investigated. The task was to bring the robot to a stable one-foot standing posture from a stable, upright initial standing posture. The machine learningbased method was also presented for analyzing behavioral preferences. The analysis results indicate that the participants of Group 2, who have a behavioral preference for random action sequences, were able to complete the task in a very short time. Conversely, the participants of Group 1, who have a behavioral preference for action sequences similar to those of previous actions required longer periods for completion. Note that the required times for task completion for the participants in Group 1 were widely ranged. Similar to simple optimization methods such as the steepest descent method, if participants (fortunately) could adopt action sequences close to the suitable sequence, the required time was short (Group 1B). If they (unfortunately) could not adopt action sequences close to the suitable sequence, the required time was very long (Group 1A). It is interesting that behavioral preferences are analogous to optimization methods. This suggests the possibility of developing a system for facilitating skill acquisition based on optimization methods. In summary, random behavioral preferences are essential for achieving higher learning efficiencies when attempting to determine unspecified suitable action sequences for completing unfamiliar tasks.
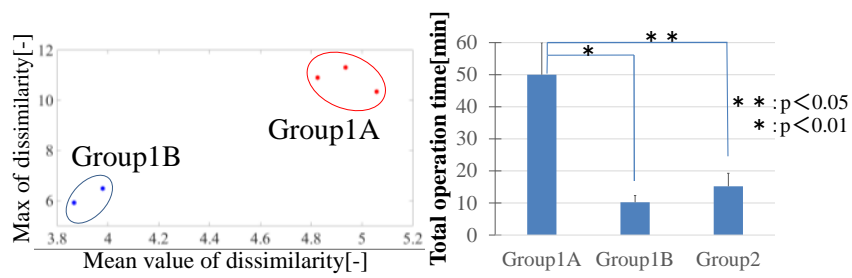

Figure 8. k-means clustering $(\mathrm{k}=2)$ Figure 9. Total operation time for result for dissimilarity between clustered groups based on suitable and trial sequences for participants in Group 1 dissimilarity between suitable and trial sequences

\section{REFERENCES}

[1] Hokuriku life care system study group, "Report for co-creation of healthy aged society utilizing ICT," JST report, 2015.

[2] K. Henmi and T. Yoshikawa, "Virtual Lesson and Its Application to Virtual Calligraphy System," Proc. of the IEEE Int. Conf. on Robotics and Automation, 1998, pp.1275-1280.

[3] Y. Yokokura, S. Katsura, K. Ohishi, "Stability Analysis and Experimental Validation of a Motion-Copying System," IEEE Trans. Industrial Electronics, vol. 56, No. 10, pp. 3906-3913, July 2009.

[4] T. Nagamatsu, Y. Kaieda, J. Kamahara, and H. Shimada, "Development of a Skill Acquisition Support System Using Expert's Eye Movement," in Human Interface and the Management of Information. Interacting in Information Environments, M. J. Smith and G. Salvendy, Ed. Springer Berlin Heidelberg, 2007, pp. 430-439.

[5] A. M. Williams and J. H. Nicola eds. Skill acquisition in sport: Research, theory and practice. Routledge, 2004.

[6] K. Lohse , D. E. Sherwood and A. F. Healy, " How changing the focus of attention affects performance, kinematics, and electromyography in dart throwing," Human Movement Science, vol.29, pp.542-555, 2010

[7] T. S. Lemieux and V. B. Penhune, "The effect of practice pattern on the acquisition, consolidation, and transfer of visual-motor sequences", Exp. Brain Res., vol.204, pp.271-281, 2010

[8] R. A. Magill and K. G. Hall, "A review of the contextual interference effect in motor skill acquisition", Human movement science, vol.9, No.3, pp. 241-289, 1990.

[9] W. B. Verwey and E. L. Abrahamse, "Distinct modes of executing movement sequences: Reacting, associating, and chunking" Acta Psychologica, Vol. 140, pp.274-282, 2014.

[10] J. A. Adams, "A closed-loop theory of motor learning," Journal of Motor Behavior, Vol. 3, pp. 111-150, 1971.

[11] W. James, The principles of psychology, New York: H. Holt \& Co., 1890.

[12] J. Paillard, "The pattern of skilled movements," in Handbook of physiology. A critical comprehensive presentation of physiological knowledge and concepts, H. W. Magoun Ed., Washington, DC: American Physiological Society, 1960, pp. 1679-1708.

[13] G. A. Miller, E. Galanter, and K. H. Pribram, Plans and the structure of behavior, New York: Holt, 1960

[14] C. M. Bishop, Pattern Recognition and Machine Learning, Springer, 2006. 